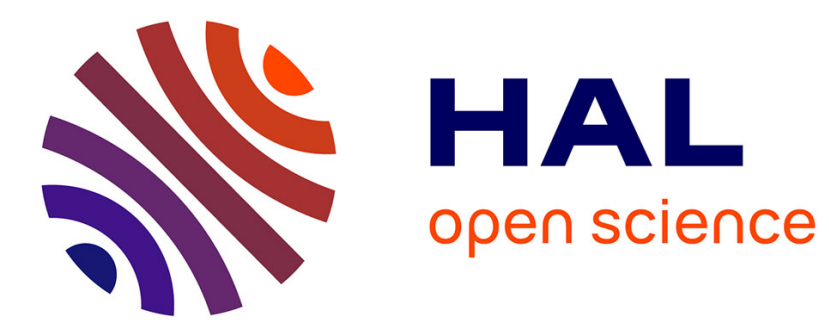

\title{
Structural studies of epitaxial BaTiO3 thin film on silicon
}

\author{
B. Wagué, J.-B. - B Brubach, G. Niu, G. Dong, L. Dai, Pascal Roy, Guillaume \\ Saint-Girons, Pedro Rojo Romeo, Y. Robach, B. Vilquin
}

\section{- To cite this version:}

B. Wagué, J.-B. - B Brubach, G. Niu, G. Dong, L. Dai, et al.. Structural studies of epitaxial BaTiO3 thin film on silicon. Thin Solid Films, 2020, 693, pp.137636. 10.1016/j.tsf.2019.137636 • hal02324723

\section{HAL Id: hal-02324723 \\ https://hal.science/hal-02324723}

Submitted on 25 Nov 2020

HAL is a multi-disciplinary open access archive for the deposit and dissemination of scientific research documents, whether they are published or not. The documents may come from teaching and research institutions in France or abroad, or from public or private research centers.
L'archive ouverte pluridisciplinaire HAL, est destinée au dépôt et à la diffusion de documents scientifiques de niveau recherche, publiés ou non, émanant des établissements d'enseignement et de recherche français ou étrangers, des laboratoires publics ou privés. 
Structural studies of epitaxial $\mathrm{BaTiO}_{3}$ thin film on silicon

B. Waguéa, J.-.B. Brubachb, G. Niuc,*, G. Dongc, L. Daic, P. Royb, G. Saint-Gironsa, P. Rojo-Romeoa, Y. Robacha, B. Vilquina,

a Universite de Lyon, Ecole Centrale de Lyon, Institut des Nanotechnologies de Lyon, CNRS UMR5270, 36 avenue Guy de Collongue, 69134 Ecully Cedex, France Synchrotron SOLEIL, AILES beamline, L'orme des merisiers, 91190 Saint Aubin, France

c Electronic Materials Research Laboratory, Key Laboratory of the Ministry of Education \& International Center for Dielectric Research, Xi'an Jiaotong University, Xi'an 710049 , China

\begin{abstract}
A B S T R A C T
BaTiO3 thin films (60 nm-thick) grown on SrTiO3/Si templates have been characterized for their structural and electrical properties. The epitaxy of the BaTiO3 film on silicon was confirmed by X-ray diffraction with good crystallinity. The temperature-dependent structural properties were checked by infrared spectroscopy in absorption mode. The films were found to remain in a single ferroelectric phase over a temperature range from 5 to $385 \mathrm{~K}$. Lowtemperature orthorhombic-rhombohedral phase transitions characteristic of bulk BaTiO3 are absent in the films due to the clamping effect from the Si substrate.
\end{abstract}




\section{Introduction}

The excellent dielectric and ferroelectric properties of barium titanate (BaTiO3, BTO) make it attractive in the field of electro-optics and nanoelectronics. As a lead-free ferroelectric ceramic, BTO is an environment- friendly material. Some of its characteristics make it a good candidate for non-volatile ferroelectric memories applications compared to other candidates $(\mathrm{Pb}(\mathrm{Zr}, \mathrm{Ti}) \mathrm{O} 3, \mathrm{SrBi} 2 \mathrm{Ta} 2 \mathrm{O} 9$, etc. $)$, such as its lower crystallization temperature, non-toxic elements, higher compatibility with integrated circuit and lower ferroelectric fatigue effects [12]. BTO can undergo three important phase transitions, with critical temperatures of $-90{ }^{\circ} \mathrm{C}, 5$ ${ }^{\circ} \mathrm{C}$ and $120{ }^{\circ} \mathrm{C}$, for the rhombohedral $\left(\mathrm{T}<-90{ }^{\circ} \mathrm{C}\right)$, orthorhombic $\left(-90{ }^{\circ} \mathrm{C}<\mathrm{T}<5{ }^{\circ} \mathrm{C}\right)$, tetragonal $\left(5^{\circ} \mathrm{C}<\mathrm{T}<120^{\circ} \mathrm{C}\right)$ and cubic phases $\left(\mathrm{T}>120^{\circ} \mathrm{C}\right)$, respectively [3]. A

room temperature, BTO exhibits a typical tetragonal crystal structure with ferroelectric properties [4]. Recently, the improvement of preparation methods and measurement techniques has led to high quality samples to study the influence of domain structures, defects, and size effects $[5,6]$. For applications, it will be necessary to integrate BTO in complementary metaloxide-semiconductor technology, which requires the use of silicon substrate. However, direct deposition of BTO on silicon is difficult due to (i) the oxidation of the silicon surface to amorphous $\mathrm{SiO} 2$ at the initial step of the growth, (ii) the interface chemical reactivity and (iii) the large lattice mismatch $(4.43 \%)$ between both materials. Several buffer layers have been utilized, including SrTiO3 (STO) [7], $\mathrm{MgO}$ [8], TiN [9], etc. In this paper, STO thin layers, which were grown by molecular beam epitaxy (MBE) on $\mathrm{Si}$ (001) substrates, were used as buffer layers. As previously mentioned, bulk BTO undergoes phase transitions. The high temperature cubic phase is paraelectric and the other phases are ferroelectric. Infrared spectroscopy is a powerful tool to study these phase transitions as the structural changes will modify the vibrational spectra [10]. In this paper, the structural and optical properties of epitaxial BTO have been studied in detail. Because the thin films suffer from defects originating from lattice and thermal coefficient expansion mismatches and clamping effects from the substrates, it is of great interest and importance to explore the possible phase transition of BTO films on Si substrate at different temperatures. This is particularly significant for future related applications such as photonic devices, non-volatile memories and negative capacitances, which use integrated BTO thin films on Si substrates. In very thin BTO films grown on STO substrates, it was reported that the films remain in the tetragonal phase with suppression of the low temperature phases present in the bulk BTO [11]. The cause of such a behavior can be the biaxial compressive strain from STO, which was also observed in BTO/STO superlattices [12]. For electro-optic applications, which require integration of very thin films on silicon - about 50-60 nm thick in a slot electro-optic waveguide [13], BTO bulk crystals exhibit a Pockels coefficient 20 times higher than in $\mathrm{LiNbO} 3$ at telecom wavelengths, making BTO an excellent material for fast and low power consumption modulators. However, Abel et al. show that the tetragonal phase implies that the material can be epitaxially grown with two different orientations depending on the process conditions and thicknesses [14]. We then study the structural characteristics of epitaxial $60 \mathrm{~nm}$-thick BTO thin film on silicon and check its phase transitions. Especially, if the film is without any transition as in [11], a good stability of its physical properties versus temperature can be expected, allowing a use of its applications in a large range of temperatures. Moreover, considering that infrared studies of thin BTO films have mostly focused on the ferroelectric phase transition from cubic to tetragonal phase above room temperature [15], we focus on the temperature range 5-385 K.

\section{Experimental conditions}

A $10 \mathrm{~nm}$-thick epitaxial STO buffer layer was firstly grown by MBE on i-Si (001) substrates. Due to its band gap of $1.1 \mathrm{eV}$, silicon is used since it is transparent in the infrared range part of the spectrum. Consequently, its absorption of the synchrotron IR radiation is negligible. An 
epitaxial $60 \mathrm{~nm}$-thick BTO thin film was then grown on STO/ Si (001) templates by radiofrequency magnetron sputtering. The detail of the epitaxy of STO on silicon can be found in [16-19]. The magnetron sputtering target was a bulk stoichiometric BaTiO3 target. The sputtering was carried out in gas ratio $\mathrm{Ar}: \mathrm{O} 2=4: 1$ at a pressure of $2 \mathrm{~Pa}$. The substrate holder was heated to a temperature of $650{ }^{\circ} \mathrm{C}$ for BTO deposition. After deposition, post-processing in a rapid thermal annealing furnace at $650{ }^{\circ} \mathrm{C}$ under an oxygen atmosphere was performed in order to reduce possible oxygen vacancies in the BTO thin film. The crystallinity of the sample was analyzed by X-ray diffraction (XRD) techniques using a 6-circles SmartLab diffractomer with a $9 \mathrm{~kW}$ rotating anode. Atomic-resolution high-angle annular dark-field (HAADF) analyses were performed using a JEM-ARM200F microscope with a probe aberration corrector, operated at $200 \mathrm{kV}$. A cross-sectional TEM/STEM specimen was prepared by standard manual grinding and thinning of the sample. Infrared absorption spectra measurements were carried out in the 30-650 cm-1 spectral range on the AILES beamline located at the Synchrotron facility SOLEIL [20,21]. The intense infrared continuum collected by the AILES beamline was focused on the entrance aperture of a high resolution IFS 125 Bruker interferometer, equipped for this experiment with a $6 \mu \mathrm{m}$ mylar-Silicon composite beam-splitter. The temperature evolution of the infrared spectra from 5 to $385 \mathrm{~K}$ was measured using $4.2 \mathrm{~K}$ cooled bolometers.

\section{Results and discussions}

At room temperature, bulk BTO is weakly tetragonal with lattice parameters $\mathrm{a}=0.3994 \mathrm{~nm}$,

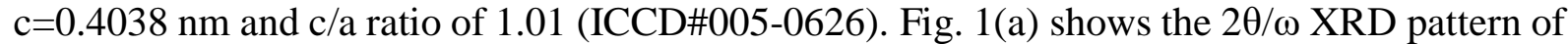
the BTO thin film deposited on STO/i-Si. Only BTO (100), BTO (200) and Si (004) peaks appear at $2=\circ \circ \theta 22.19,45.27$ and $69.14^{\circ}$, respectively, leading to an out-of-plane parameter value of BTO of $0.4003 \mathrm{~nm} \pm 0.0001 \mathrm{~nm}$. From the rocking curve shown in Fig. 1(c), the mosaicity of the BTO layer can be evaluated as $1.4^{\circ}$ from $\omega$ scan, which is in good agreement with the literature [22-24] and indicates the good crystallinity of the grown BTO film. Fig. 1(d) shows the reciprocal space map (RSM) of BTO (200) Bragg reflection and in this figure, only one spot of BTO (200) can be observed, which confirms that BTO is single a-domain. The STO (002) reflection is not observed on the RSM due to its small thickness. $\emptyset$-scan of $360^{\circ}$ around (101) plane of i-Si and BTO was performed and the results were shown in Fig. 1(b). The shift by $45^{\circ}$ of the four peaks from one material to the peaks of the other material indicated that the BTO layer was grown epitaxially on i-Si thanks to the thin STO buffer layer. Since the BTO film is in the tetragonal phase, the in-plane parameter was calculated from the 2-theta values of the BTO 100 reflection (Fig. 1a) and BTO 101 reflection (Fig. 1b) and is equal to 0.4042 $\mathrm{nm} \pm 0.0001 \mathrm{~nm}$. The film has therefore a tetragonal structure with an a/c ratio of 1.01 . The BTO film is a-axis oriented on the silicon substrate. In the case of the BTO epitaxial film grown on silicon, the thickness has a strong effect on the film orientation. Indeed, for thinner films, lattice parameters reported by various groups in the range 7-8 $\mathrm{nm}$ show full c-axis orientation, with an out-of-plane lattice parameter close to the bulk c value [23-25]. Thicker films of 80-130 nm films grown on a few nm thick STO template show full relaxation with 001 out-of-plane orientation and lattice parameters $a=0.400 \mathrm{~nm}$ and $c=4.030 \mathrm{~nm}(\mathrm{a} / \mathrm{c}=1.01) \quad[14,26,27]$. Our film with a thickness of $60 \mathrm{~nm}$ shows similar characteristics to very thin films. The atomic arrangement and epitaxial relationship between the BTO thin film and the substrate is visualized by transmission electron microscopy (TEM) for the BTO/STO/Si heterostructure, as shown in Fig. 2. Fig. 2(a) and (b) show the cross-sectional views in high resolution TEM (HRTEM) and high-angle annular dark-field imaging (HAADF) scanning TEM (STEM) modes, respectively. It can be seen that BTO crystallizes well and a thin amorphous layer is formed between the silicon and the STO (Fig. 2a and b). It was previously shown [17-19] that such a type of amorphous layer of $\mathrm{SiO} 2$ is formed due to the penetration of oxygen passing through the growth front and its reaction with Si during the epitaxial growth of films under high oxygen partial 
pressure, which breaks the original abrupt feature between STO and Si. From selected area electron diffraction (SAED) (Fig. 2c), the epitaxial relationship is determined as tetragonal perovskite (001)//silicon (110), i.e. the perovskite orientation is rotated in-plane by $45^{\circ}$ with respect to the silicon crystal. Only a single series of BTO spots can be observed, demonstrating that the BTO is mono-oriented on Si (001). The SAED pattern measured at $200 \mathrm{keV}$ allowed also to estimate the lattice parameters of the BTO film, with a good agreement with the previous extracted values from the XRD figures. Fig. 2(d) shows an example of an edge dislocation in the BTO layer at the BTO/STO interface. The strain is reduced by the formation of such dislocations at the BTO/STO interface in order to minimize the energy of the films $[14,26]$. The BTO layer grown on the STO/i-Si seed layer is then partly relaxed.

Both in the ferroelectric and the paraelectric phases, BTO in bulk has one molecule with five atoms per unit cell, which results in 12 optical modes. We have performed IR absorption spectra measurements of BTO in the range from 85 to $650 \mathrm{~cm}-1$. Room temperature measurements were carried out to observe the difference in vibrational frequency along the polar a-axis. The spectrum measured with polarized infrared radiation at room temperature is shown in Fig. 3. The most intense lines observed in the polarized infrared spectrum are at 165, 288, 307, 480 and $517 \mathrm{~cm}-1$ and are attributed to A1/E (LO), A1(TO), E(TO+LO)/B1, E(TO), A1(TO) bulk phonons respectively [28-31] and indicated by dash lines on the Fig. 3. The peak at $307 \mathrm{~cm}-1$ is due to mixed LO2-TO3 phonon of E symmetry. Good indicators of BTO tetragonal phase at room temperature are the presence of the $\mathrm{E}(\mathrm{TO}+\mathrm{LO})$ overdamped soft mode and existence of a very narrow peak at $307 \mathrm{~cm}-1$, which vanishes in the paraelectric cubic phase. The high frequency $\mathrm{E}$ mode around $480 \mathrm{~cm}-1$ is weak even in the bulk material [28-29]. In the film, all peaks are broad, due to the strains generated by the substrate, and overlap with the broad peak at $517 \mathrm{~cm}-1$. The temperature evolution of BTO thin film was carried out in a range from 5 to $385 \mathrm{~K}$. The temperature dependence of the spectra shown in Fig. 4 does not show sharp changes in this range. When the temperature increases, the phonon mode lines broaden. An examination of these results shows that the temperature dependent behavior of the BTO film is qualitatively similar to that observed in [30]. However the wavenumbers of their maxima are almost constant and do not show any shift, even if the signal is noisy. An examination of these results shows that the temperature dependent behavior of the BTO film is qualitatively similar to that observed in [30]. This behavior seems to indicate that the BTO thin film does not undergo any phase transition in the temperature range 5-385 K. Indeed, phase transitions corresponding to orthorhombic and rhombohedral phases of the bulk appear to be absent in the $60 \mathrm{~nm}$-thick epitaxial BTO film grown on silicon substrate with STO buffer layer. There are however some important differences between the spectra behavior in the thin film. Unlike the bulk case in which absorption peaks can vanish and/or peaks wavenumber can shift at the transition temperature [31], in our film, the spectra intensity does not show any abrupt modification at any temperature and any shift of the phonons wavenumber is observed with the temperature variation. The same behavior was observed for thin films grown both on STO and LaAlO3 substrates [11,12,32]. Additional characterizations are underway to check in particular the influence of the BTO film thickness on the phase transitions. The out-of-plane a-axis and the highly insulating behavior of the i-Si substrate, used as bottom electrode, avoid the measurement of any ferroelectric loops on the tetragonal BTO film since the polarization is inplane oriented. Nevertheless, ferroelectricity can be expected in our BTO film since this property was reported from electrical or electromechanical measurements in such similar BTO layers on silicon [25].

\section{$\underline{\text { 4. Conclusion }}$}

X-ray diffraction and infrared spectroscopy were used to study the ferroelectric phase transition of epitaxial BTO layers deposited on the silicon substrate with a $10 \mathrm{~nm}$-thick STO buffer layer. 
The $60 \mathrm{~nm}$-thick BTO film has a tetragonal phase and is a-axis out-of-plane oriented. The temperature variation of bulk BTO phase transitions as like orthorhombic- rhombohedral transition are missing in the film which stays in the tetragonal phase at whatever temperature in our experiment. Generally, the larger lattice mismatch between BTO and Si $(\sim 4.0 \%)$ compared to that of STO and Si $(\sim 1.7 \%)$ makes it more difficult to obtain barium titanate films of high structural quality on Si substrates. Thanks to the BTO elastic modulus of $120 \mathrm{GPa}$ [33] and its average thermal expansion coefficient of $10-5 / \mathrm{K}$ [34] close to that of $2.10-6 / \mathrm{K}$ for silicon, the BTO thin film is clamped on the silicon and the film then follows the expansion of the silicon substrate without any of its own phase transition.

\section{Acknowledgments}

This work was realized on the Nanolyon technology platform and at Synchrotron SOLEIL. We thank "ARC4 Energy", "Region Rhone-Alpes", Natural Science Foundation of China (Grant No. 51602247), Natural Science Fundamental research Project of Shaanxi province of China (No. 2017JQ6003), and Synchrotron SOLEIL for their financial support.

\section{$\underline{\text { References }}$}

[1] S.R. Shannigrahi, H.M. Jang, Fatigue-free lead zirconate titanate-based capacitors for nonvolatile memories, Appl. Phys. Lett. 79 (2001) 1051.

[2] S.T. Zhang, B. Yang, Y.F. Chen, Z.G. Liu, X.B. Yin, Y. Wang, M. Wang, N. Ben Ming, SrBi4Ti4O15 thin films and their ferroelectric fatigue behaviors under varying switching pulse widths and frequencies, J. Appl. Phys. 91 (2002) 3160.

[3] Y.V. Kolenko, K.A. Kovnir, I.S. Neira, T. Taniguchi, T. Ishigaki, T. Watanabe, N. Sakamoto, M. Yoshimura, A novel, controlled, and high-yield solvothermal drying route to nanosized barium titanate powders, J. Phys. Chem. C. 111 (2007) 7306. [4] L. Qi, B.I. Lee, P. Badheka, D.H. Yoon, W.D. Samuels, G.J. Exarhos, Short-range dissolution-precipitation crystallization of hydrothermal barium titanate, J. Eur. Ceram. Soc. 24 (2004) 3553.

[5] S. Wada, T. Suzuki, M. Osada, M. Kakihana, T. Noma, Change of macroscopic and microscopic symmetry of barium titanate single crystal around curie temperature, Jpn. J. Appl. Phys. 37 (1998) 5385.

[6] G. Tarrach, P.L. Lagos, R.Z. Hermans, F. Schlaphof, C. Loppacher, L.M. Eng, Nanometer spot allocation for Raman spectroscopy on ferroelectrics by polarization and piezoresponse force microscopy, Appl. Phys. Lett. 79 (2001) 3152.

[7] F. Amy, A. Wan, A. Kahn, F.J. Walker, R.A. McKee, Surface and interface chemical composition of thin epitaxial SrTiO3 and BaTiO3 films: Photoemission investigation, J. Appl. Phys. 96 (2004) 1601.

[8] A.R. Meier, F. Niu, B.W. Wessels, Integration of $\mathrm{BaTiO} 3$ on Si (0 0 1) using $\mathrm{MgO} / \mathrm{STO}$ buffer layers by molecular beam epitaxy, J. Cryst. Growth. 294 (2006) 401.

[9] N. Shu, A. Kumar, M.R. Alam, H.L. Chan, Q. You, Study of dielectric properties of laser processed BaTiO3 thin films on Si 100 / with TiN buffer layer, Appl. Surf. Sci. 109 (1997) 366.

[10] W. Peng, R. Tétot, G. Niu, E. Amzallag, B. Vilquin, P. Roy, Room-temperature soft mode and ferroelectric like polarization in SrTiO3 ultrathin films : infrared and ab initio study, Sci. Rep. 7 (2017) 2160.

[11] D.A. Tenne, P. Turner, J.D. Schmidt, M. Biegalski, Y.L. Li, L.Q. Chen, A. Soukiassian, S. Trolier-McKinstry, D.G. Schlom, X.X. Xi, D.D. Fong, P.H. Fuoss, J.A. Eastman, G.B. Stephenson, C. Thompson, S.K. Streiffer, Ferroelectricity in ultrathin BaTiO3 films: probing the size effect by ultraviolet raman spectroscopy, Phys. Rev. Lett. 103 (2009) 177601. 
[12] D.A. Tenne, A. Bruchhausen, N.D. Lanzillotti-Kimura, A. Fainstein, R.S. Katiyar, A. Cantarero, A. Soukiassian, V. Vaithyanathan, J.H. Haeni, W. Tian, D.G. Schlom, K.J. Choi, D.M. Kim, C.B. Eom, H.P. Sun, X.Q. Pan, Y.L. Li, Long-qing Chen, Q.X. Jia, S.M.

Nakhmanson, Probing nanoscale ferroelectricity by ultraviolet raman spectroscopy, Science 313 (2006) 1614.

[13] Alvaro Rosa, Domenico Tulli, Pau Castera, Ana M Gutierrez, Amadeu Griol, Mariano Baquero, Bertrand Vilquin, Felix Eltes, Stefan Abel, Jean Fompeyrine, Pablo Sanchis, Barium titanate (BaTiO3) RF characterization for application in electro-optic modulators, Opt. Mater. Express 7 (2017) 4328.

[14] S. Abel, M. Sousa, C. Rossel, D. Caimi, M.D. Rossell, R. Erni, J. Fompeyrine, C. Marchiori, Controlling tetragonality and crystalline orientation in $\mathrm{BaTiO}_{3}$ nanolayers grown on Si, Nanotechnology 24 (2013) 285701.

[15] M.B. Smith, K. Page, T. Siegrist, P.L. Redmond, E.C. Walter, R. Seshadri, L.E. Brus, M.L. Steigerwald, S. Barbara, B. Laboratories, A.V Mountain, M. Hill, Crystal structure and the paraelectric-to-ferroelectric phase transition of nanoscale BaTiO3, J. Am. Chem. Soc. (2008) 6955.

[16] G. Niu, G. Saint-Girons, B. Vilquin, G. Delhaye, J.L. Maurice, C. Botella, Y. Robach, G. Hollinger, Molecular beam epitaxy of SrTiO3 on Si (001): early stages of the growth and strain relaxation, Appl. Phys. Lett. 95 (2009) 062902.

[17] G. Niu, J. Penuelas, L. Largeau, B. Vilquin, J.-L. Maurice, C. Botella, G. Hollinger, Guillaume Saint-Girons, Evidence for the formation of two phases during the growth of SrTiO3 on silicon, Phys. Rev. BA 292 (83) (2011) 054105.

[18] G. Niu, B. Vilquin, J. Penuelas, C. Botella, G. Hollinger, G. Saint-Girons, Heteroepitaxy of SrTiO3 thin films on $\mathrm{Si}(001)$ using different growth strategies: toward substrate-like quality, J. Vac. Sci. Technol. B 29 (2011) 041207.

[19] G. Niu, W.W. Peng, G. Saint-Girons, J. Penuelas, P. Roy, J.-B. Brubach, J.- L. Maurice, G. Hollinger, B. Vilquin, Direct epitaxial growth of SrTiO3 on Si (001): interface, crystallization and IR evidence of phase transition, Thin Solid Films 519 (2011) 5722. [20] P. Roy, M. Rouzieres, Z. Qi, O. Chubar, The AILES Infrared Beamline on the third generation Synchrotron Radiation Facility SOLEIL, Infrared Phys. Technol. 49 (2006) 139.

[21] J.B. Brubach, L. Manceron, M. Rouzières, O. Pirali, D. Balcon, F.K. Tchana, V. Boudon, M. Tudorie, T. Huet, A. Cuisset, P. Roy, Performance of the AILES THzinfrared beamline at SOLEIL for high resolution spectroscopy, AIP Conf. Proc. 1214 (2010) 81.

[22] W.J. Lin, T.Y. Tseng, H.B. Lu, S.L. Tu, S.J. Yang, I.N. Lin, Growth and ferroelectricity of epitaxial-like BaTiO3 films on single-crystal $\mathrm{MgO}, \mathrm{SrTiO} 3$, and silicon substrates synthesized by pulsed laser deposition, J. Appl. Phys. 77 (1995) 6466.

[23] L. Mazet, S.M. Yang, S.V Kalinin, A review of molecular beam epitaxy of ferroelectric BaTiO3 films on Si, Ge and GaAs substrates and their applications, Sci. Technol. Adv. Mater. 16 (2015) 36005.

[24] G. Niu, S. Yin, G. Saint-Girons, B. Gautier, P. Lecoeur, V. Pillard, G. Hollinger, B. Vilquin, Epitaxy of BaTiO3 thin film on Si(001) using a SrTiO3 buffer layer for non-volatile memory application, Microelectron. Eng. 88 (2011) 1232.

[25] C. Dubourdieu, John Bruley, Thomas M. Arruda, Agham Posadas, Jean Jordan- Sweet, Martin M. Frank, Eduard Cartier, David J. Frank, Sergei V. Kalinin, Alexander A. Demkov, Vijay Narayanan, Switching of ferroelectric polarization in epitaxial BaTiO3 films on silicon without a conducting bottom electrode, Nat. Nanotechnol. 8 (2013) 748.

[26] S. Abel, Thilo Stöferle, Chiara Marchiori, Christophe Rossel, Marta D. Rossell, Rolf Erni, Daniele Caimi, Marilyne Sousa, Alexei Chelnokov, Bert J. Offrein, Jean Fompeyrine, Strong electro-optically active lead-free ferroelectric integrated on silicon, Nat. Commun. 4 (2013) 1671. 
[27] C. Xiong, W.H.P. Pernice, J.H. Ngai, J.W. Reiner, D Kumah, F.J. Walker, C.H. Ahn, H.X. Tang, Active silicon integrated nanophotonics: ferroelectric BaTiO3 devices, Nano Lett. 14 (2014) 1419.

[28] Scalabrain, A.S. Craves, D.S. Shima, S.P.S. Porto, Temperature Dependence of the A, and E Optical Phonons in BaTiO3, Phys., Status Solidi 79 (1977) 731.

[29] J.A. Sanjurjo, R.S. Katiyar, S.P.S. Porto, Temperature dependence of dipolar modes in ferroelectric BaTi03 by infrared studies, Phys. Rev. B. 22 (1980) 2396. [30] M. El Marssi, F. Le Marrec, I.A. Lukyanchuk, M.G. Karkut, Ferroelectric transition in an epitaxial barium titanate thin film: Raman spectroscopy and X-ray diffraction study, J. Appl. Phys. 94 (2003) 3307.

[31] C.H. Perry, D.B. Hall, Temperature dependence of the Raman spectrum of BaTiO3, Phys. Rev. Lett. 15 (1965) 700.

[32] D.A. Tenne, X.X. Xi, Y.L. Li, L.Q. Chen, A. Soukiassian, M.H. Zhu, A.R. James, J. Lettieri, D.G. Schlom, W. Tian, X.Q. Pan, Absence of low-temperature phase transitions in epitaxial BaTiO3 thin films, Phys. Rev. B 69 (2004) 2.

[33] A.C. Dent, C.R. Bowen, R. Stevens, M.G. Cain, M. Stewart, Effective elastic properties for unpoled barium titanate, J. Eur. Ceram. Soc. 27 (2007) 3739.

[34] M. Siegert, J.G. Lisoni, C.H. Lei, A. Eckau, W. Zander, C.L. Jia, J. Schubert, C. Buchal, Epitaxial BaTiO3 thin films on different substrates for optical waveguide applications, Mater. Res. Soc. Symp. - Proc. 597 (2000) 145.

\section{Figures}
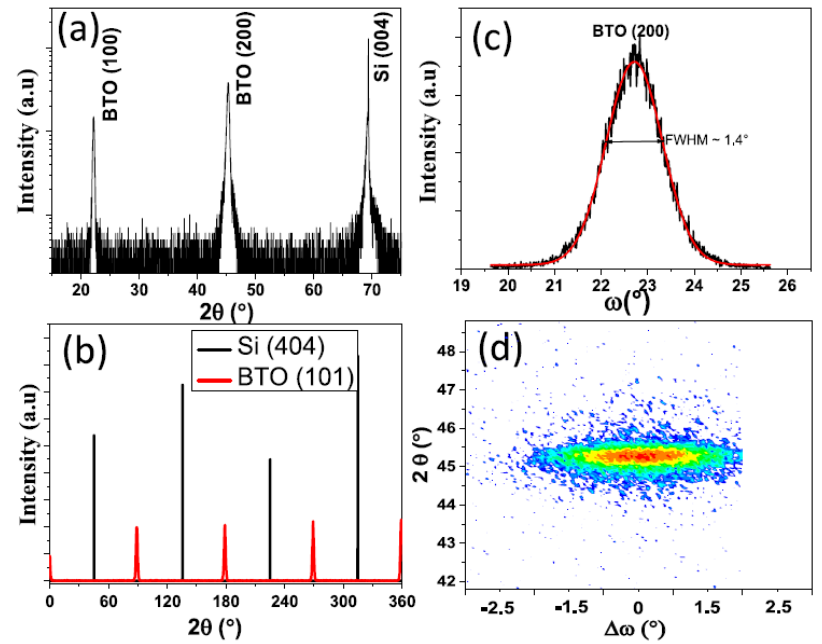

Fig. 1. (a) XRD 20/ $\omega$ diffratogram of the epitaxial BTO reflection for $60 \mathrm{~nm}-\mathrm{BTO} / 10 \mathrm{~nm}-\mathrm{STO} / \mathrm{Si}$ (001) sample, (b) Ø-scan of $360^{\circ}$ around (101) plane of $i$-Si and BTO, (c) rocking curve of BTO (200) in $\omega$ Bragg condition with $F W H M \approx 1.4^{\circ}$, (d) reciprocal space map of BTO (200) reflection. 

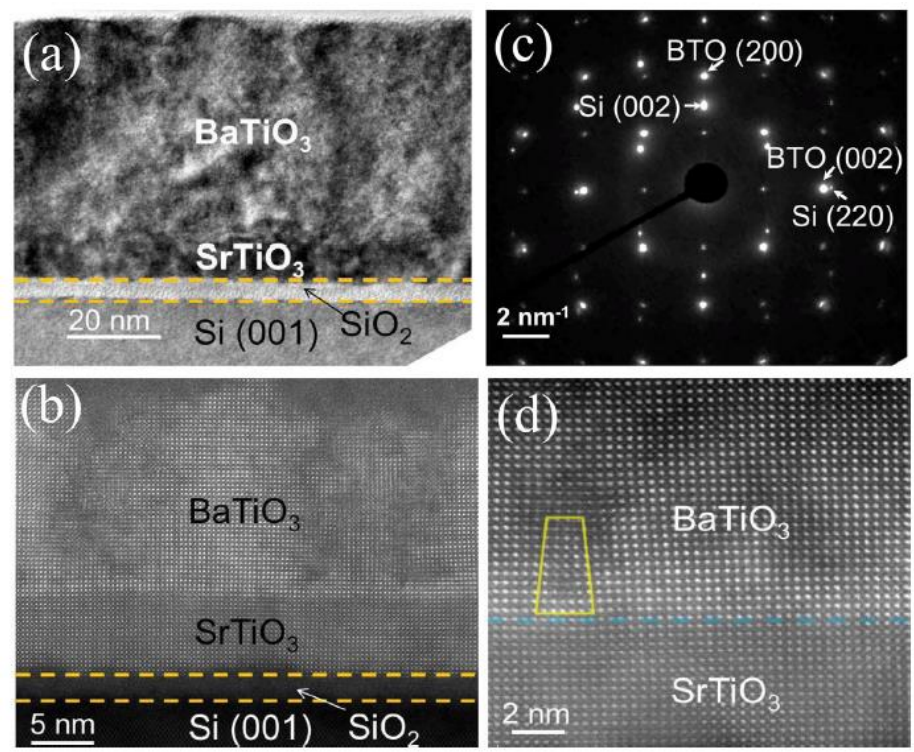

Fig. 2. Cross-sectional view of BTO/STO/Si heterostructure: (a) HRTEM and (b) HAADF STEM images, (c) The SAED image showing the epitaxial relationship between BTO and the silicon substrate and $(d)$ formation of dislocations at the BTO/STO interface.

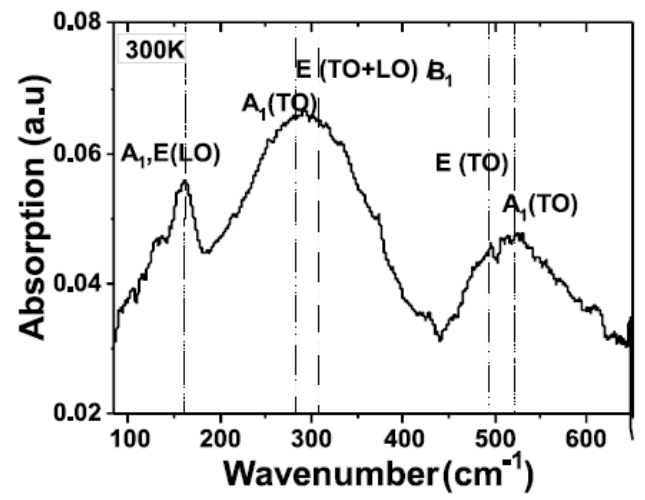

Fig. 3. Room temperature infrared absorption spectrum of the BTO thin film grown on silicon with STO buffer layer.

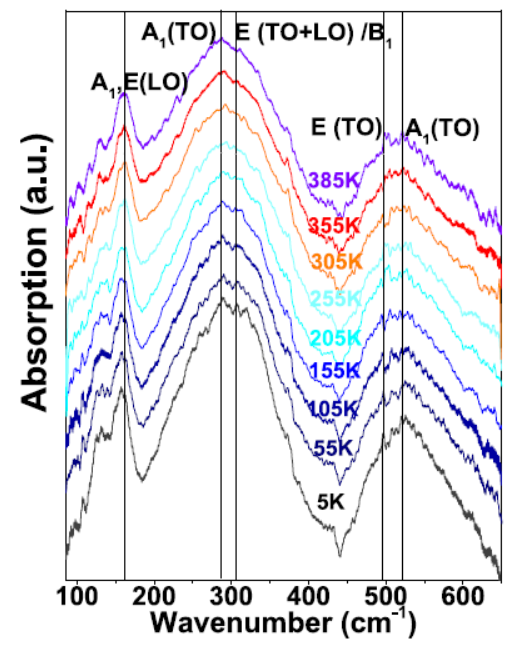

Fig. 4. Evolution of the infrared absorption spectra with the temperature from $5 \mathrm{~K}$ to $385 \mathrm{~K}$. 